\title{
Why E-Cigarettes and Vaping Probably Increase Risk for Lung Diseases, Heart Attacks, Coronary Artery Disease, Stroke and Death: Potential Roles of Marijuana, Thiocyanate and Unrecognized Magnesium Deficiency; A Hypothesis
}

\author{
Burton M Altura ${ }^{1-6 *}$, Asefa Gebrewold ${ }^{1}$, Anthony Carella ${ }^{1}$, Aimin Zhang ${ }^{1}$, Wenyan Li $^{1}$, Bella T Altura ${ }^{1,3-6}$ \\ ${ }^{1}$ Department of Physiology and Pharmacology, The State University of New York Downstate Medical Center, Brooklyn, New York, USA \\ ${ }^{2}$ Department of Medicine, The State University of New York Downstate Medical Center, Brooklyn, New York, USA \\ ${ }^{3}$ The Center for Cardiovascular and Muscle Research, The State University of New York Downstate Medical Center, Brooklyn, New York, USA \\ ${ }^{4}$ The School for Graduate Studies in Cellular and Molecular Biology, The State University of New York Downstate Medical Center, Brooklyn, \\ New York, USA \\ ${ }^{5}$ Bio-Defense Systems, Inc., Rockville Centre, New York, USA \\ ${ }^{6}$ Orient Biomedica, Estero, Florida; Magnesium for Health Foundation, Patterson, California, USA \\ *Corresponding Author: Burton M Altura, Professor, Department of Physiology and Pharmacology, The State University of New York \\ Downstate Medical Center, Brooklyn, New York, USA.
}

Received: October 15, 2019; Published: October 16, 2019

DOI: $10.31080 /$ ASPS.2019.03.0421

It is now estimated that 1 out of 20 Americans may indulge in using E-cigarettes and vaping [1]. New research obtained by the CDC and published in several very recent editions of Morbidity and Mortality Weekly Reports links E-cigarette-vaping to high risks for heart attacks ( $>50 \%)$, coronary artery disease $(>60 \%)$, lung diseases $(>60 \%)$, strokes $(>70 \%)$ and death [1-6]. This scourge now appears to be focused, primarily (i.e., more than $65 \%$ of all subjects), on youths 16-24 years of age. These multistate USA outbreaks, in particular with lung diseases have appeared, so far as of this writing, in almost 1,300 people with almost 30 deaths. Although the specific chemical (s) responsible for these outbreaks are not as yet known, we believe the focus should be on nicotine, marijuana-cannabis products, thiocyanate, and hypomagnesemia. Below, we posit why we think marijuana products (particularly delta- 9 tetrahydrocannabinol), thiocyanate and hypomagnesemia should be centers of focus.

Recent findings on almost twenty people suggest that vaping results in lungs which seem like they have been exposed to toxic chemicals

Workers at The Mayo Clinic have reported that the lungs of almost 20 vapers (two of which had died) had lung tissues that resembled chemical burns [7]. They have suggested these lung tissues looked very similar to what has been observed in industrial accidents [7].

Recent Evidence Demonstrates that Marijuana-cannabis Products and Thiocyanates Cause Vasospasm and Inflammation of Coronary, Pulmonary and Cerebral Blood Vessels and Deplete Vascular Smooth Muscle Cells of Magnesium Ions.

Several studies (since 1990), using isolated blood vessels (including peripheral, coronary, pulmonary and cerebral), have now shown that sodium and potassium thiocyanates [8-13], as well as marijuana products (e.g., delta-9 tetrahydrocannabinol) [14], cause vascular smooth muscle cells to contract (and often spasm) when exposed to these substances. Moreover, when primary cells from these diverse vessels are cultured in-vitro (using techniques developed in our labs), for several days they: 1 . Lose free intracellular magnesium ions ([ $\left.\mathrm{Mg}^{2+}\right] \mathrm{I}$ [15], and 2. Demonstrate an increase in TNF-alpha, IL-1, and IL-6 as well as activation of cellular NF-kB [18], signs characteristic of the beginnings of an inflammatory response. 
Why E-Cigarettes and Vaping Probably Increase Risk for Lung Diseases, Heart Attacks, Coronary Artery Disease, Stroke and Death: Potential Roles of Marijuana, Thiocyanate and Unrecognized Magnesium Deficiency; A Hypothesis

Potential significance of vascular and inflammatory actions of marijuana-cannabis products and thiocyanates to risks for heart attacks, lung diseases, coronary artery disease, strokes and death

Approximately 50 years ago, we first demonstrated that when isolated macro- and micro-blood vessels (including peripheral, coronary, pulmonary, umbilical-placental and cerebral) are exposed to low extracellular $\mathrm{Mg}^{2+}$, the vascular smooth muscle cells undergo contractions and vasospasm with changes in their geometrical shapes and calcium overload [16-24]. We suggested that such findings, if verified in human subjects might portend a rationale for ischemic heart disease (IHD), angina, pulmonary artery and lung diseases, coronary artery diseases CAD), strokes, hypertension, preeclampsia, and sudden-death ischemic heart disease (SDIHD) [20-22,24-27]. Several lines of evidence have now been brought forth in numerous experimental and human studies from different parts of the globe which support our hypothesis [28-33].

At the turn of the $20^{\text {th }}$ century, people in North America and Europe were ingesting about 450-550 $\mathrm{mg}$ of $\mathrm{Mg} / \mathrm{day}$, whereas now people living in these areas are now only ingesting about 165-238 mg of Mg/day [24,26,34,35]. Thus, more than $75 \%$ of most North Americans and Europeans are Mg deficient.

If vaping causes depletion of intracellular $\mathrm{Mg}^{2+}$ in pulmonary, cerebral and coronary blood vessels, as we suspect, and additional depletion of $\left[\mathrm{Mg}^{2+}\right] \mathrm{I}$ from inhaling thiocyanates and marijuana-cannabis products takes place, again as we suspect, then a constellation of pathological effects would take place, over time, as indicated above. It should be pointed out, here, that many of the blood vessels and cardiac tissues from the victims of vaping-induced deaths appear to exhibit muscle cells in various stages of cell death. Recently, we have reported results from Mg-deficient animals which indicate coronary vascular smooth and cardiac muscle cells are found in different stages of programmed cell death (i.e., apoptosis; necroptosis; pyroptosis; or ferroptosis) [36-39]. Whether or not any of these forms of programmed cell death are present in users of e-cigarettes remains to be determined. It is, however, possible if our hypothesis is correct, that intravenous administration of $\mathrm{Mg}$ salts, followed by oral dosing, might be therapeutically-helpful in prophylactic and therapeutic treatment against the pathological actions of vaping.

\section{Conclusions and Future Thoughts}

As of this writing almost 1,300 people (with approximately 30 deaths), in the USA, have presented with various cardiovascular and lung ailments after using E-cigarettes and "vaping". More than $65 \%$ of the subjects are youths between 18-25 years of age. Why vaping causes high risks for development of lung diseases, coronary artery disease, heart attacks, strokes, and death is not known. Most all of the victims were found to have high levels of delta 9-tetrahydrocannabinol and had to have had elevated levels of thiocyanate. Based on our findings with diverse vascular smooth muscles, and primary cell cultures, we have posited a new hypothesis that marijuana-cannabis products and thiocyanates, by depleting blood vessels of Mg along with nicotine, found in many E-cigarettes, are probably instrumental in causing some or all of the pathological effects of vaping. As most North Americans and Europeans (particularly youths) have diets deficient in $\mathrm{Mg}$, such a situation would tend to exacerbate the pathological effects of vaping.

In view of our findings, and suggestions, we believe clinical trials and investigative studies should be undertaken to determine whether: 1 . Pulmonary and coronary tissues of e-cigarette smokers have decreased levels of intracellular free $\mathrm{Mg}$; and 2. Intravenous $\mathrm{Mg}$ compounds given to E-cigarette -vapor users would yield potential prophylactic and therapeutic effects.

\section{Acknowledgements}

Many of our laboratory and clinical investigations have been supported by research grants from different branches of the $\mathrm{Na}$ tional Institutes of Health (i.e., The National Heart, Lung and Blood Institute; The National Institute on Drug Abuse; The National Institute on Alcoholism and Alcohol Abuse; and The National Mental Health Institute) awarded to B.M.A. and B.T.A. While many of our studies were still in progress, our dear forensic colleague, Anthony Carella, passed away. His expertise will be sorely missed.

\section{Bibliography}

1. Napoli N. "E-cigarettes linked to heart attacks, coronary artery disease and depression". American College of Cardiology, Wash, DC (2019).

2. Davidson K., et al. "Outbreak of e-cigarette associated acute lipoid pneumonia -North Carolina, July-August 2019”. Morbidity and Mortality Weekly Report 68 (2019): 784-786. 
Why E-Cigarettes and Vaping Probably Increase Risk for Lung Diseases, Heart Attacks, Coronary Artery Disease, Stroke and Death: Potential Roles of Marijuana, Thiocyanate and Unrecognized Magnesium Deficiency; A Hypothesis

3. Scler JG., et al. "Severe pulmonary disease associated with electronic-cigarette-product use-Interim guidance". Morbidity and Mortality Weekly Report 68 (2019): 787-790.

4. Ghinau I., et al. E-cigarette product use or vaping, among persons with associated lung injury-----Illinois and Wisconsin, April -September 2019. Morbidity and Mortality Weekly Report 68 (2019).

5. Perrine CG., et al. "Characteristics of a multistate outbreak of lung injury associated with E-cigarette use, or vapingUnites States, 2019". Morbidity and Mortality Weekly Report 68 (2019).

6. Tompson D. Vaping tied to rise in stroke, heart attack risk (2019).

7. Grady D. "Lung damage from vaping resembles chemical burns, report says". The New York Times (2019).

8. Zhang AM., et al. "SCN- ions induce contractions of vascular muscle from male but not female rats". European Journal of Pharmacology 179 (1990): 287-294.

9. Zhang AM., et al. "Unusual effects of SCN and lyotropic anions on contractility of vascular smooth muscle from female rats". Naunyn-Schmiedeberg's Archives of Pharmacology 344 (1991): 193-200.

10. Standley PR., et al. "Effects of SCN substitution for Cl- on tension, $\{\mathrm{Ca} 2+] \mathrm{I}$, and ionic currents in vascular smooth muscle". Life Science 59 (1996): 739-752.

11. Engstrom KG and Sehlin J. "Thiocyanate induces vasoconstriction in rat-tail artery primed with norepinephrine". Journal of Laboratory and Clinical Medicine 127 (1996): 391-400.

12. Li W., et al. "Magnesium modulates contractile responses of rat aorta to thiocyanate: A possible relationship to smokinginduced atherosclerosis". Toxicology and Applied Pharmacology 157 (1999): 77-84.

13. Prusa AM and Plass CA. "Melatonin attenuates thiocyanateinduced vasoconstriction in aortic rings". Saudi Pharmacology 25 (2017): 993-998.

14. Altura BM., et al. "Increased risk of stroke using marijuanacannabis products: Evidence for dangerous effects on brain circulation and the unrecognized roles of magnesium". Drugs and Alcohol Addiction 1 (2018): 001-006.
15. Altura BM., et al. Thiocyanates and tetrahydrocannabinol produce loss of Mg2+, increased synthesis of cytokines, and activation of NF-kB in piglet primary coronary and pulmonary vascular muscles cells in culture: Potential relationship to pathological actions of e-cigarette smoking and vaping (2019).

16. Altura BM and Altura BT. "Influence of magnesium on drug-induced contractions and on ion content in rabbit aorta". American Journal of Physiology 220 (1971): 938-944.

17. Altura BM and Altura BT. "Magnesium and contraction of arterial smooth muscle". Microvascular Research 7 (1974): 145155.

18. Altura BM and Altura BT. "Extracellular magnesium and contraction of vascular smooth muscle". In: Excitation-Contraction Coupling of Smooth Muscle, Casteels R, Godfraind T, Ruegg JC, eds. North-Holland Publ. Co., Amsterdam (1977): 137-144.

19. Altura BM and Altura BT. "Magnesium and vascular tone and reactivity". Blood Vessels 15 (1978): 5-16.

20. Turlapaty PDMV and Altura BM. "Magnesium deficiency produces spasms of coronary arteries: relationship to suddendeath ischemic heart disease". Science 208 (1980): 198-200.

21. Altura BM., et al. "Magnesium deficiency -induced spasms of umbilical vessels: relation to preeclampsia, hypertension, growth retardation". Science 221 (1983): 376-378.

22. Altura BM., et al. "Magnesium deficiency and hypertension: correlation between magnesium-deficient diets and microcirculatory changes in situ". Science 223 (1984): 1315-1317.

23. Zhang A., et al. "Magnesium regulates intracellular free ionized calcium concentration and cell geometry in vascular smooth muscle cells". Biochimica et Biophysica Acta 1134 (1992): 2529.

24. Altura BM., et al. "Can hypomagnesemia put the squeeze on coronary arteries: An unappreciated factor in myocardial ischemia, heart attacks and sudden cardiac death". EC Orthopaedics 10 (2019): 572-581.

25. Altura BM and Altura BT. "Magnesium ions and contraction of vascular smooth muscle: Relationship to vascular diseases". Federation Proc 40 (1981): 2672-2679.

26. Altura BM and Altura BT. "Magnesium and cardiovascular biology: An important link between cardiovascular risk factors and atherogenesis". Cellular and Molecular Biology Research 41 (1995): 347-359. 
27. Altura BM., et al. "The expression of platelet-activating factor is induced by low extracellular Mg2+ in aortic, cerebral and neonatal coronary vascular smooth muscle: Cross-talk with ceramide production, NF-kB and proto-oncogenes: Possible links to atherogenesis and sudden cardiac death in children and infants and aging: Hypothesis, review and viewpoint". International Journal of Cardiovascular Research 3 (2016): 4767.

28. Kimura T., et al. "Effects of magnesium on the tone of isolated human coronary arteries: Comparison with diltiazem and nitroglycerin". Circulation 79 (1989): 1118-1124.

29. Goto K., et al. "Magnesium deficiency detected by intravenous loading test in variant angina pectoris". American Journal of Cardiology 65 (1990): 709-712.

30. Simko P. "Pathophysiological aspects of the protective effect of magnesium in myocardial infarction (review)". Acta medica Hungarica 50 (1994): 55-64.

31. Satake K., et al. "Relation between severity of magnesium deficiency and frequency of anginal attacks in men with variant angina". Journal of the American College of Cardiology 28 (1996): 897-902.

32. Sueda S., et al. "Magnesium deficiency in patients with myocardial infarction and provoked by coronary artery spasm". Japanese Circulation Journal 65 (2001): 643-648.

33. Minato N., et al. "Perioperative coronary artery spasm in offpump coronary bypass grafting and its possible relation with perioperative hypomagnesemia". Annals of Thoracic and Cardiovascular Surgery 12 (2006): 32-36.

34. Mosfegh A., et al. What We Eat in Americ. NHANES 20052006: usual Nutrient Intakes from Food and Water Compared to 1997 Dietary Reference Intakes for Vitamin D, Calcium, Phosphorus, and Magnesium. U.S. Department of Agricultural Research (2009).

35. NHANES 2009-2012. "Dietary Reference Intakes for Vitamin D, Calcium, Phosphorus, and Magnesium". U.S. Department of Agricultural Research (2016).

36. Altura BM., et al. "Short-term magnesium deficiency results in decreased levels of serum sphingomyelin, lipid peroxidation, and apoptosis". The American Journal of Physiology-Heart and Circulatory Physiology 297 (2009): H86-H92.
37. Altura BM., et al. "Regulated RIPK3 necroptosis is produced in cardiovascular tissues and cells in dietary magnesium deficiency: Roles of cytokines and their potential importance in inflammation and atherogenesis". Journal of Medical and Surgical Pathology 2 (2017).

38. Altura BM., et al. "Regulated ferroptosis cell death is produced in cardiovascular tissues and cells in dietary magnesium deficiency: Initiation of roles of glutathione, mitochondrial alterations and lipid peroxidation in inflammation and atherogenesis". EC Pharmacology and Toxicology 6 (2018): 535-541.

39. Altura BM., et al. "Regulated pyroptosis is produced in cardiovascular tissues and cells in dietary magnesium deficiency: Cross-talk with cytokines, PAF, telomerases and aging". EC Pharmacology and Toxicology (2019).

\section{Volume 2 Issue 11 November 2019 (C) All rights are reserved by Burton M Altura.}

